Weniger Sex

\title{
Nach einer KHK-Diagnose vergeht vielen Männern die Lust
}

\author{
Männer über 50 mit koronarer Herzkrankheit haben seltener Sex als Gleichaltrige mit \\ unauffälligen Koronarien. Das gilt besonders für die Zeit kurz nach der Diagnose, wie eine \\ englische Studie ergeben hat.
}

\begin{abstract}
— „Sex ist ein zentraler Bestandteil intimer Beziehungen" - mit diesem Satz beginnt die Zusammenfassung der Studienergebnisse, die Andrew Steptoe vom University College London zusammen mit Kollegen zum Thema "Sex und KHK" vorgelegt hat. Gewiss hat die Wissenschaft schon spektakulärere Erkenntnisse zutage gefördert. Doch der Punkt, auf den die englischen Forscher hinauswollen, ist ein anderer: Wenn Patienten an KHK erkranken, könnte es sein - so die Überlegung -, dass neben der Herzauch die Sexualfunktion beeinträchtigt ist. Weil es dann mit besagtem zentralem Bestandteil hapert, könnte Beziehungsstress aufkommen. Und Stress ist gefährlich fürs ohnehin angegriffene Herz.
\end{abstract}

\section{Krankes Herz - schlechter Sex?}

Steptoe und Mitarbeiter haben deshalb untersucht, wie es um das Liebesleben älterer Herrschaften über 50, die an einer KHK erkrankt sind, bestellt ist. Sie analysierten dafür Querschnittsdaten von rund 3.000 Männern und 3.700 Frauen, die an der English Longitudinal Study of Ageing beteiligt waren. 376 Männer und 279 Frauen hatten eine KHK-Diagnose erhalten. Dabei zeigte sich: Eine KHK hat durchaus Einfluss auf das Sexualleben. Allerdings hält er sich in Grenzen.

Im summarischen Vergleich von Frauen über 50 mit und ohne KHK war überhaupt kein statistisch relevanter Unterschied in den sexuellen Aktivitäten festzustellen. Das mag auch daran liegen, dass Frauen keine Erektionsstörungen bekommen können. Diese nämlich markierten bei den Männern mit und ohne

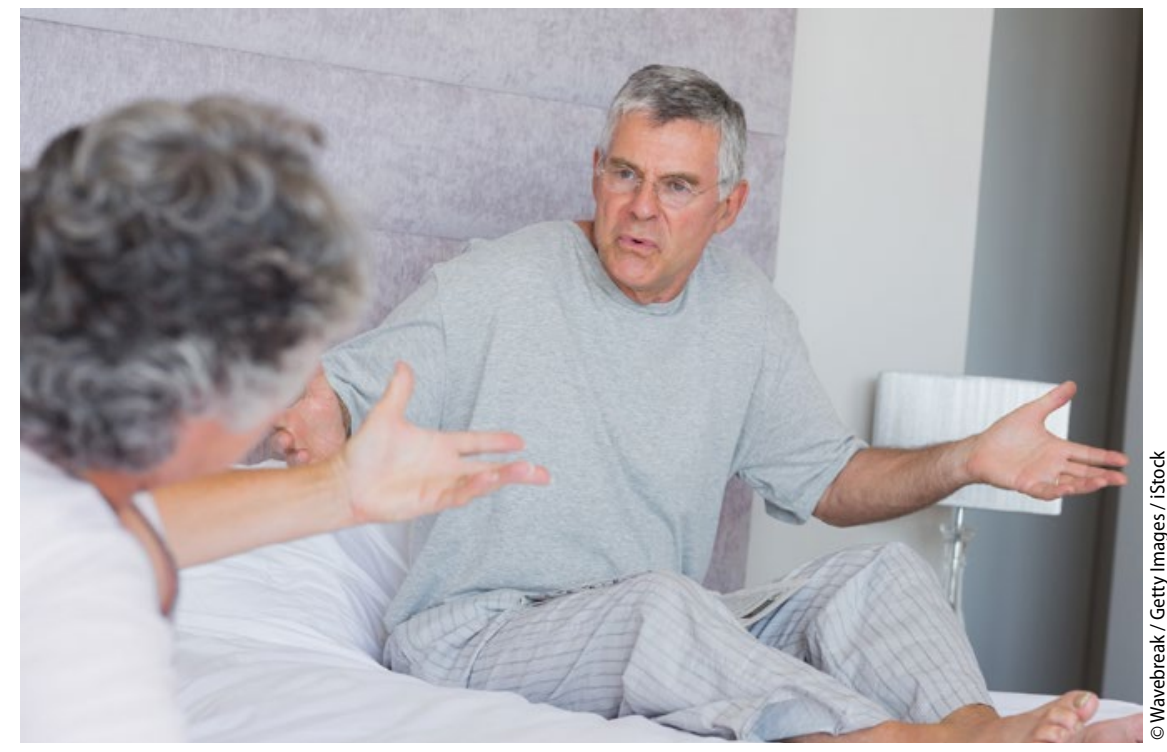

Erst eine KHK bescheinigt bekommen, dann auch noch Beziehungsstress ...

KHK einen deutlich signifikanten Unterschied ( $47 \%$ vs. $38 \%)$. Männer mit KHK hatten auch seltener Sex als die Vergleichsmänner: Der Anteil der Aktiven lag bei $69 \%$ und damit niedriger als bei herzgesunden Männern (80\%), aber immer noch höher als bei den herzgesunden Frauen (56\%). Und Männer mit KHK hatten nicht nur weniger Sex, sie dachten auch seltener daran (75\% vs. $82 \%)$.

Damit sind dann aber alle Unterschiede aufgezählt, die sich in den Betten von KHK-Patienten und jenen von Personen ohne KHK auftun. Darüber hinaus treten diese Phänomene nur bei Patienten in den ersten vier Jahren nach der KHK-Diagnose prominent auf. Liegt die Diagnose länger zurück, sind keine signifikanten Differenzen festzustellen.

Steptoe und Kollegen untersuchten zudem, ob die Medikation die Sexual- funktion beeinflusst. Negativ auf die Erektion wirkten Diuretika und Statine, dabei könne es sich aber um Zufallsergebnisse handeln, so die Wissenschaftler. Für andere Medikamentenklassen waren keine Effekte nachweisbar.

Die Forscher um Steptoe raten, KHKPatienten in Sachen Sex gut zu beraten, damit sie ihren Partnern zum Nutzen der Beziehung rascher wieder nahezukommen wagen. Hier gilt als Faustregel: Wer zügig spazieren gehen bzw. die Treppen von zwei Stockwerken erklimmen kann, ist auch fit genug für den zentralen Bestandteil intimer Beziehungen.

- Dr. Robert Bublak

- Quelle: Steptoe A et al. Sexual activity and concerns in people with coronary heart disease from a population-based study. Heart 2016, online 10. Juni; doi: 10.1136/ heartjnl-2015-308993 